\title{
Intestinal transcriptomes in Kazakh sheep with different haplotypes after experimental Echinococcus granulosus infection
}

\author{
Xin $\mathrm{Li}^{1,2, a}$, Song Jiang ${ }^{2}$, Xuhai Wang ${ }^{2}$, and Bin $\mathrm{Jia}^{2, *}$ \\ ${ }^{1}$ College of Life Sciences, Shihezi University, Road Beisi, Shihezi 832003, Xinjiang, PR China \\ ${ }^{2}$ College of Animal Science and Technology, Shihezi University, Road Beisi, Shihezi 832003, Xinjiang, PR China
}

Received 8 October 2020, Accepted 4 February 2021, Published online 5 March 2021

\begin{abstract}
Cystic echinococcosis (CE) is a chronic zoonosis caused by infection with the larval stage of the cestode Echinococcus granulosus. As the intermediate host, sheep are highly susceptible to this disease. Our previous studies have shown that sheep with haplotype MHC Mva Ibc-Sac IIab-Hin1I ab were resistant to CE infection, while their counterparts without this haplotype were not. In order to reveal the molecular mechanism of resistance in Kazakh sheep, after selecting the differential miRNA in our previous study, herein, transcriptome analyses were conducted to detect the differential expression genes in the intestinal tissue of Kazakh sheep with resistant and non-resistant MHC haplotypes, after peroral infection with E. granulosus eggs. A total of 3835 differentially expressed genes were identified between the two groups, with 2229 upregulated and 1606 downregulated. Further function analysis showed that the most significant genes were related to both innate immune response and adaptive response participating in the defense against $E$. granulosus infection and the metabolic changes associated with it. The results suggest that genes related to lectin receptors, NK cells activation, chemokines, and tumor necrosis factor, may play important roles in the response of intestinal tissue to E. granulosus.
\end{abstract}

Key words: Differentially expressed genes, Disease resistance, Echinococcus granulosus, Intestinal tissue, Transcriptome analysis.

Résumé - Transcriptomes intestinaux chez des moutons kazakhs de différents haplotypes après une infection expérimentale avec Echinococcus granulosus. L'échinococcose kystique (EK) est une zoonose chronique qui est causée par une infection au stade larvaire du cestode Echinococcus granulosus. En tant qu'hôte intermédiaire, les moutons sont très sensibles à cette maladie. Nos études précédentes ont montré que les moutons avec l'haplotype MHC Mva Ibc-Sac IIab-Hin1I ab étaient résistants à l'EK, alors que leurs homologues sans cet haplotype ne l'étaient pas. Afin de révéler le mécanisme moléculaire de la résistance chez le mouton kazakh, après avoir sélectionné le miARN différentiel dans notre étude précédente, des analyses de transcriptome ont été menées dans ce travail pour détecter les gènes d'expression différentielle dans le tissu intestinal de mouton kazakh avec des haplotypes MHC résistants et non résistants, après une infection pérorale par des œufs d'E. granulosus. Un total de 3835 gènes différentiellement exprimés ont été identifiés entre les deux groupes, avec 2229 régulés à la hausse et 1606 à la baisse. Une analyse fonctionnelle plus poussée a montré que les gènes les plus significatifs étaient liés à la fois à la réponse immunitaire innée et à la réponse adaptative participant à la défense contre l'infection à $E$. granulosus et aux changements métaboliques qui y étaient associés. Les résultats suggèrent que les gènes liés aux récepteurs de la lectine, à l'activation des cellules NK, aux chimiokines et au facteur de nécrose tumorale peuvent jouer un rôle important dans la réponse du tissu intestinal à E. granulosus.

\section{Introduction}

Cystic echinococcosis (CE), a zoonotic disease caused by Echinococcus granulosus, poses a great threat to the health of humans and domestic animals [51]. The life cycle of

* Corresponding author: 215273439@qq. com

${ }^{a}$ Institutes 1 and 2 contributed equally.
E. granulosus is complex and involves two hosts: definitive and intermediate. Infection with E. granulosus occurs after oral ingestion of infective eggs (an oncosphere containing the invasive hexacanth [9]). The hexacanth then hatches in the small intestine of the intermediate host, penetrates the mucosal tissue, enters the blood circulation system, and migrates to various organs (e.g. the liver and lungs), where it develop cysts filled 
with fluid and protoscoleces [6]. However, in definitive hosts like dogs, adult parasites develop from the protoscolex in the intestine. In domestic animals, especially in sheep, which appear to be highly susceptible to infection [38], CE causes considerable health problems and thereby significantly affects the income of herders [32].

Echinococcus granulosus infection in the host is a complex dynamic process, which involves the recognition and interaction of a variety of biological molecules, including genes [33], proteins [1], and miRNAs [42]. Upregulation or downregulation of molecular expression likely causes different individuals of the same host to have different resistance, similarly to reports about Plasmodium homocircumflexum [17]. It is very important to understand the interaction mechanisms between the host and the parasite. Research and discussions about these mechanisms will help to determine the key factors of immune regulation in the host from the perspective of molecular biology, and further explore the mechanism of disease resistance. This research will also provide new ideas for mining molecular markers of disease resistance and directional genetic breeding [19].

Previously, we carried out a series of studies to research the relationship between MHC polymorphisms and resistance or susceptibility to CE in native Chinese sheep, including Chinese Merino [21], Duolang [28], and Kazakh sheep [29]. Our previous studies indicated that in Kazakh sheep, when exposed to the same number of parasites, the rate of $E$. granulosus infection in the internal organs of sheep with haplotype $M H C M v a \mathrm{I}$ bc-SacII ab-Hin1I ab haplotype was significantly lower than in sheep without this haplotype. Statistical results and analysis showed that sheep with this haplotype have an increased genetic capability to respond to and subsequently reject E. granulosus when infected. The haplotype MHC MvaI bc-SacII ab-Hin1I ab was therefore identified as a genetic marker of CE resistance in Kazakh sheep [29].

Afterwards, studies were conducted to reveal the mechanism of CE resistance in Kazakh sheep, and most of our research was focused on intestinal tissue. It has been found that when sheep were infected with oncospheres (larvae of E. granulosus), the small intestine tissue was the first mucosal immune barrier that must be passed through, where various antibodies, cytokines, and other immune molecules are expressed in response to exogenous stimuli [12]. These molecules could be involved in stopping larval growth of E. granulosus at the very first stages [11, 21, 28, 29]. Hence, we were interested to see the molecular mechanisms of CE resistance in Kazakh sheep with the resistant haplotype, at the very first stage of infection. Against this background, we previously performed microRNA sequencing, and found several microRNAs in the intestinal tissue, which were related to the inflammatory pathway and played important roles in CE resistance in sheep [22]. Since microRNAs exert their functions by regulating the expression of target genes, we were curious to see whether genes related to inflammation and immune response were varied in these sheep, including innate immune cells activation, chemokines, and tumor necrosis factor. It is, therefore, necessary to conduct genome-wide characterization of transcriptome profiles involved in resistance to $\mathrm{CE}$ infection in sheep, especially in sheep with different CE resistance.
Transcriptome sequencing aims at the set of all transcripts, including mRNAs, rRNA, tRNA, and other noncoding RNAs, produced in one or a population of cells in a specific developmental stage or physiological condition [13]. In the present study, using high-throughput transcriptome sequencing, we screened a number of differential genes in the small intestinal tissue of Kazakh sheep after infection with E. granulosus. In terms of their functions, we concluded that innate immune response plays the first defense role in CE resistance in sheep. In addition, adaptive immune response played an important role in defense against $E$. granulosus infection. Moreover, our study revealed that metabolic changes contribute to defense against E. granulosus infection. These findings laid the foundation for a discussion of the molecular mechanism of Kazakh sheep resistance to E. granulosus.

\section{Materials and methods}

\section{Ethics statement}

This study was performed in strict compliance with the recommendations of the National Institutes of Health guideline for the care and use of laboratory animals (NIH Publication No. 8023, revised 1978), and the Animal Care and Use Committee of Shihezi University approved all procedures and experiments (Approval No. A2018-138-01). Local strain dogs and Kazakh sheep were purchased from farmers around Shihezi city. The farmers gave oral consent for the use of their animals in this research after they were provided with explanations about the purpose of the research and on efforts to ensure the welfare of animals.

\section{Animals and experimental design}

The preparation method of the experimental materials was similar to previous research reports [22]. Since no studies have shown that the CE resistance of sheep is related to sex, 2-yearold Kazakh ewes with CE-resistant [MvaI bc-SacII ab-Hin1I ab (R)] and CE-non-resistant [Mvalbc-SacIIab-Hin1Iab (NR)] haplotypes were selected, as described previously, and raised on the farm of Shihezi University. All animals were raised under the same conditions of free access to water and food, in natural lighting. They were divided into two groups according to their MHC haplotype: three sheep with the CE-resistant haplotype were referred to as the CE-R group, three sheep with the CE-non-resistant haplotype constituted the CE-NR group, and a single sheep with the CE-non-resistant haplotype formed the CK (Control Check) group. All the sheep were screened for the absence of hydatid cysts and were healthy prior to the experiment. In line with the previous approach, all the sheep were orally infected with E. granulosus eggs. Since our previous research showed that there were already significant differences in miRNAs in the small intestine at $8 \mathrm{~h}$ after infection, we speculated that differential gene expression may occur at the same time point. As a result, all animals were sacrificed $8 \mathrm{~h}$ after infection. Immediately afterward, the intestinal tissue of every sheep was removed. Approximately $100-\mathrm{mm}^{3}$-sized intestinal tissue blocks were collected and frozen immediately 
in liquid nitrogen prior to long-term storage at $-80{ }^{\circ} \mathrm{C}$ until RNA extraction.

\section{Experimental pipeline of RNA-seq and quality control}

Total RNA was isolated from every sample using TRIZOL reagent, and divided into two parts, one for RNA-seq, and the other for real-time PCR to verify the expression of genes detected by RNA-seq.

During the RNA-seq process, after DNase I treatment, magnetic beads with Oligo (dT) were used to isolate mRNA. Mixed with the fragmentation buffer, the mRNA was fragmented into short fragments. Then, cDNA was synthesized using mRNA fragments as templates. cDNA fragments were purified and resolved with EB buffer for end reparation and adenine addition. After that, the fragments were connected to adapters. After agarose gel electrophoresis, the suitable fragments were selected for the polymerase chain reaction (PCR) amplification as templates. During the quality control (QC) steps, an Agilent 2100 Bioanalyzer and ABI StepOnePlus Real-Time PCR System were used for the quantification and qualification of the sample library. In this process, the intestinal tissue of every sheep in the CE-R group was prepared to an individual paired-end library for RNA-seq, like in the CE-NR group. Each library was sequenced using Illumina HiSeq 2000 (BGI, Huada Genomics Institute, Shenzhen, China).

Primary sequencing data produced using Illumina HiSeq 2000 , called raw reads, were filtered with the SOAPnuke program (version 1.5.0) [27] by (1) removing reads containing sequencing adapters; (2) removing reads whose low quality base ratio (base quality less than or equal to 5) was more than $20 \%$; and (3) removing reads whose unknown base ("N" base) ratio was more than $5 \%$, after clean reads were obtained and stored in FASTQ format. After the above quality control (QC) step, raw reads were filtered into clean reads. Because the CE-R group contained the clean data from three individual libraries (for three samples), these data were combined to form the clean data of the CE-R group, and were aligned to the reference genome with SOAPaligner/SOAP2 [26], like in the CENR group. In this search, the sheep oar3.1 database of livestock genomics was selected as the reference genome (http://www. livestockgenomics.csiro.au/sheep/oar3.1.php/).

The clean data were used to calculate the distribution of reads on reference genes and perform coverage analysis. Gene coverage was calculated as the percentage of a gene covered by reads. This value is equal to the ratio of the base number in a gene covered by unique mapping reads to the total base number of that gene by the SAMtools program.

\section{Normalization of expressed genes}

There are many different patterns to normalize the gene expression level. In this search, we used the RPKM method [36] (Reads per kilobase transcriptome per million mapped reads), because it is able to eliminate the influence of different gene lengths and sequencing discrepancy on the calculation of gene expression. Therefore, the calculated results can be directly used to compare the differences in gene expression between samples.

\section{Screening and verification of differentially expressed genes (DEGs)}

After the alignment result passed QC, according to the significance of digital gene expression profiles which were reported on genome research by Audio [2], only genes with a value of FDR $\leq 0.001$ and $\mid \log 2$ ratio $\mid \geq 1$ were selected as DEGs. Since DEG analysis generated a large number of problems in which thousands of hypotheses (is gene $x$ differentially expressed between the two groups) were tested simultaneously, correction for false-positive (type I errors) and false-negative (type II) errors was performed using the False Discovery Rate (FDR) method [4]. We assumed that we picked out $R$ differentially expressed genes in which $\mathrm{S}$ genes actually showed differential expression and the other $V$ genes were false positives. As we decided that the error ratio " $Q=V / R$ " must stay below a cutoff, we preset the FDR to a number no larger than 0.001 .

In addition, before transcriptome functional annotation, nine differentially expressed genes were randomly selected to conduct real-time PCR analysis to verify the expression of genes detected by RNA sequence. The GAPDH gene was used as an internal control (for PCR verifications only). Primer sequences are listed in Table 1. The mRNA expression levels for all samples were normalized to the level for GAPDH housekeeping genes. The expression level in every sample group was expressed by the mean \pm standard deviation $(x \pm s)$. The data were analyzed by SPSS17.0 software.

\section{Functional annotation}

Gene Ontology (GO) enrichment analysis provides all GO terms that significantly enriched in a list of DEGs, compared to a genome background, and filter the DEGs that correspond to specific biological functions. All DEGs between two groups were mapped to GO terms in the database (http://www.geneontology.org/), calculating gene numbers for every term. We then used hypergeometric tests to find significantly enriched GO terms in the input list of DEGs, based on the GO:TermFinder program (http://smd.stanford.edu/help/GO-TermFinder/ GO_TermFinder_help.shtml/). GO terms fulfilling a threshold of $p$-value $\leq 0.05$ were defined as significantly enriched GO terms in DEGs. This analysis was able to recognize the main biological functions that DEGs exercise.

Functional annotations of the DEGs were conducted using the Blast2GO program against the public the NCBI nr database. The Kyoto Encyclopedia of Genes and Genomes (KEGG, http://www.genome.jp/kegg/) library was used to classify and group these identified DEGs.

\section{Results}

In the present study, firstly, RNA-sequence analysis showed that 46,934,632 reads were obtained in the CE-R group, $76.60 \%$ of which matched the reference genome. The number 
Table 1. Primer sequences for qRT-PCR analysis of gene transcripts.

\begin{tabular}{|c|c|c|c|}
\hline Gene & Primer sequences $\left(5^{\prime}-3^{\prime}\right)$ & Expected size (bp) & Annealing temperature $\left({ }^{\circ} \mathrm{C}\right)$ \\
\hline \multirow[t]{2}{*}{ NTNG1 } & F: CAAAGACAGGTTCGCATT & 214 & 59.5 \\
\hline & R: CCTTCCGTGCACTTTTAT & & \\
\hline \multirow[t]{2}{*}{ EphA3 } & F: CCAGCGATGTATGGAGTTA & 180 & 58.5 \\
\hline & R: CTTTCTGCCAGCAGTCTAG & & \\
\hline \multirow[t]{2}{*}{ NCOA1 } & F: GATCTAACCTCCCTTCTGCTTTT & 228 & 61 \\
\hline & R: GGAACAGAGAGGACAACGCA & & \\
\hline \multirow[t]{2}{*}{$S C D$} & F: CGCTGGCACATCAACTTTACC & 124 & 56.6 \\
\hline & R: TTTCCTCTCCAGTTCTTTTCATCC & & \\
\hline \multirow[t]{2}{*}{ GHR } & F: AGGTTGCTCAGCCACAAA & 281 & 58.9 \\
\hline & R: TGGGGAAAGGACCACATT & & \\
\hline \multirow[t]{2}{*}{ SMAD3 } & F: CGACTACAGCCATTCCATCC & 120 & 60.5 \\
\hline & R: TGGTCACTGGTCTCTCCATCT & & \\
\hline \multirow[t]{2}{*}{ TGFBRI } & F: ATTCTGTGGCTTTGCCTGAA & 175 & 60 \\
\hline & R: GCAGTTTCCTGGGTCTGAAG & & \\
\hline \multirow[t]{2}{*}{$I G F 1$} & F: GACAGGAATCGTGGATGAGTG & 270 & 57 \\
\hline & R: AACAGGTAACTCGTGCAGAGC & & \\
\hline \multirow[t]{2}{*}{$L P L$} & F: GTCATCGTGGTGGACTGG & 398 & 59.3 \\
\hline & R: TGGAAAGTGCCTCCGTTA & & \\
\hline \multirow[t]{2}{*}{ GAPDH } & F: CTGACCTGCCGCCTGGAGAAA & 149 & 59.0 \\
\hline & R: GTAGAAGAGTGAGTGTCGCTGTT & & \\
\hline
\end{tabular}

Table 2. Alignment statistics of reads and reference genome.

\begin{tabular}{lccc}
\hline Alignment types & CE-R & CE-NR & CK \\
\hline Total reads & 46934632 & 47203918 & 47241022 \\
Total base pairs & 4224116880 & 4248352620 & 4251691980 \\
Total mapped reads & $35950168(76.60 \%)$ & $38570247(81.71 \%)$ & $35662569(75.49 \%)$ \\
Perfect matched reads & $24082846(51.31 \%)$ & $24897763(52.75 \%)$ & $21930554(46.42 \%)$ \\
$\leq 5$ bp Mismatched reads & $11867322(25.28 \%)$ & $13672484(28.96 \%)$ & $13732015(29.07 \%)$ \\
Unique match & $30693890(65.40 \%)$ & $27695773(58.67 \%)$ & $28046058(59.37 \%)$ \\
Multi-position match & $5256278(11.20 \%)$ & $10874474(23.04 \%)$ & $7616511(16.12 \%)$ \\
Total unmapped reads & $10984464(23.40 \%)$ & $8633671(18.29 \%)$ & $11578453(24.51 \%)$ \\
\hline
\end{tabular}

of unique match reads was 30,693,890, accounting for $65.40 \%$ of the total reads. Similarly, 47,203,918 reads were obtained in the CE-NR group, with $81.71 \%$ matching to the reference genome. The number of unique match reads was 27,695,773, accounting for $58.67 \%$ of the total. Detailed information is listed in Table 2.

Results showed that 16,346 genes were detected in CE-R group samples, while 16,038 were detected in the CE-NR group (Fig. 1A). Furthermore, the analysis of DEGs showed 3835 genes which were different in the CE-R group compared with CE-NR group, of which 2229 were upregulated and 1606 were downregulated. The Venn diagrams of DEGs between groups are shown in Figures 1B and 1C. Scatter charts of detected genes are shown in Figure 2, where the $X$-axis and $Y$-axis present two samples $\log 2$ value of expression, red (up) and green (down) dots mean the gene has significant differences (FDR $\leq 0.001$, two fold difference), and the blue dot means no significant difference. A heatmap highlights the patterns of expression for many DEGs between two sample groups (Fig. 3).

In summary, GO functional clustering and KEGG pathway analysis showed that various DEGs related to immune response were significantly more highly expressed in the CE-R group than in the CE-NR group. Firstly, we found that some genes related to lectin receptors, including CLEC9A, CLEC10A, and CLEC4G were significantly more highly expressed in the CE-R group than in the CE-NR group. Secondly, we found that a large number of DEGs related to NK cell activation (KLRC2, IRAK1, FCRL6, and $N K G 7)$, mast cell activation ( $M C P I$ and $I R C P I)$, and T cells were significantly more highly expressed in the CE-R group than in the CE-NR group. Additionally, genes related to chemokines (CXCL12, CXCL14, CCLA, CCL5, CCL11, CCL25, and CCL28), compensatory inflammation repair mechanisms (CYP17A1, CYP1A1, CYP2C19, and CYP3A24), and tumor necrosis factor (TNFRSF13B, TNFRSF17, TNFRSF1A, TNFRSF4, TNFSF10, TNFSF13, TNFSF15, TNFSF18) were more highly expressed in the CE-resistant group than in the CEnon-resistant sheep (Table 3). In addition, some genes related to MHC were also significantly more highly expressed in the CE$\mathrm{R}$ group than in the CE-NR group, including LOC101103957, LOC101108696, LOC101109747, LOC101120148, LOC10 1109080, LOC101105609 and LOC101107908.

Furthermore, nine genes with a differential expression level were randomly selected to conduct real-time PCR validation of the transcriptome analysis. The results from real-time PCR are shown in Figure 4, and strongly correlated with those generated from transcriptome analysis. 

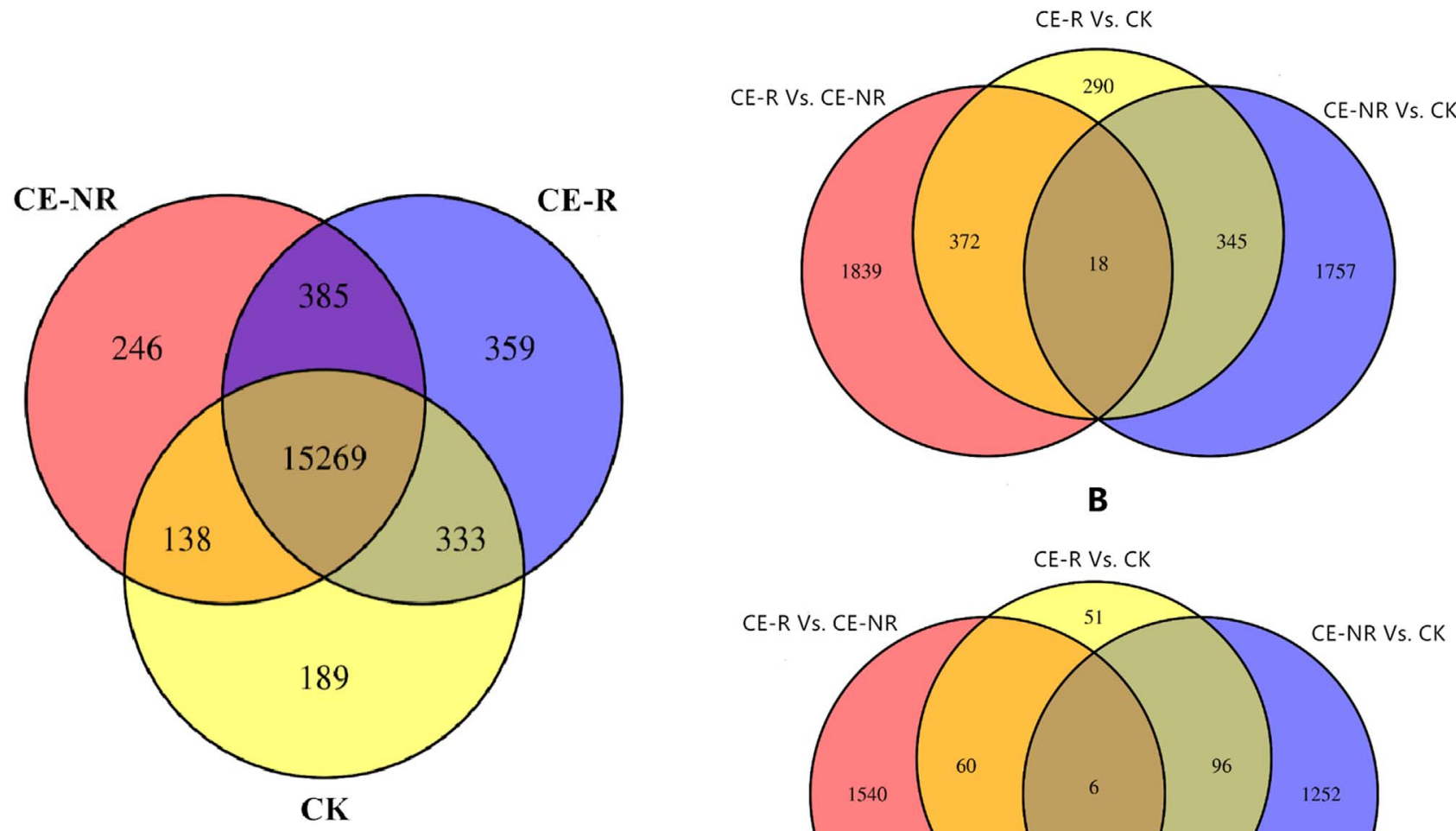

B

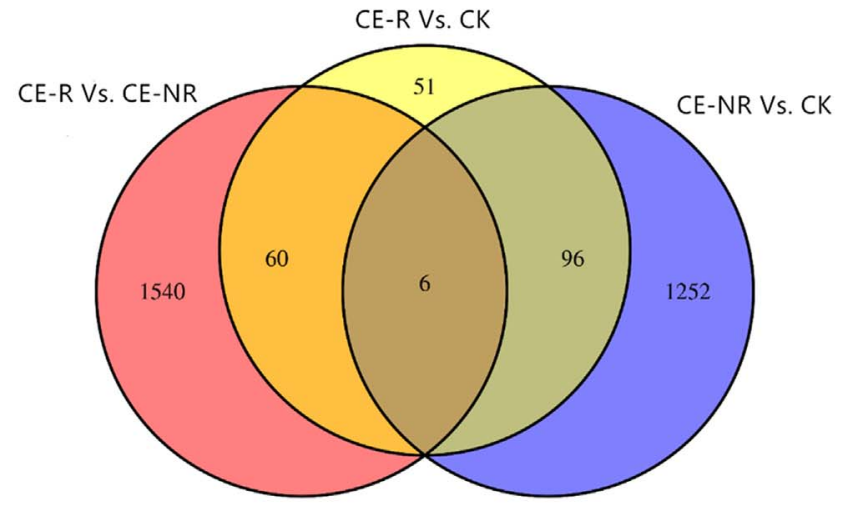

C

Figure 1. Venn diagram of all genes and differential expression genes (DEGs) between groups. Figure 1A shows all identified genes in each group. Figure 1B shows the upregulated DEGs between groups, while Figure 1C shows the downregulated DEGs between groups.
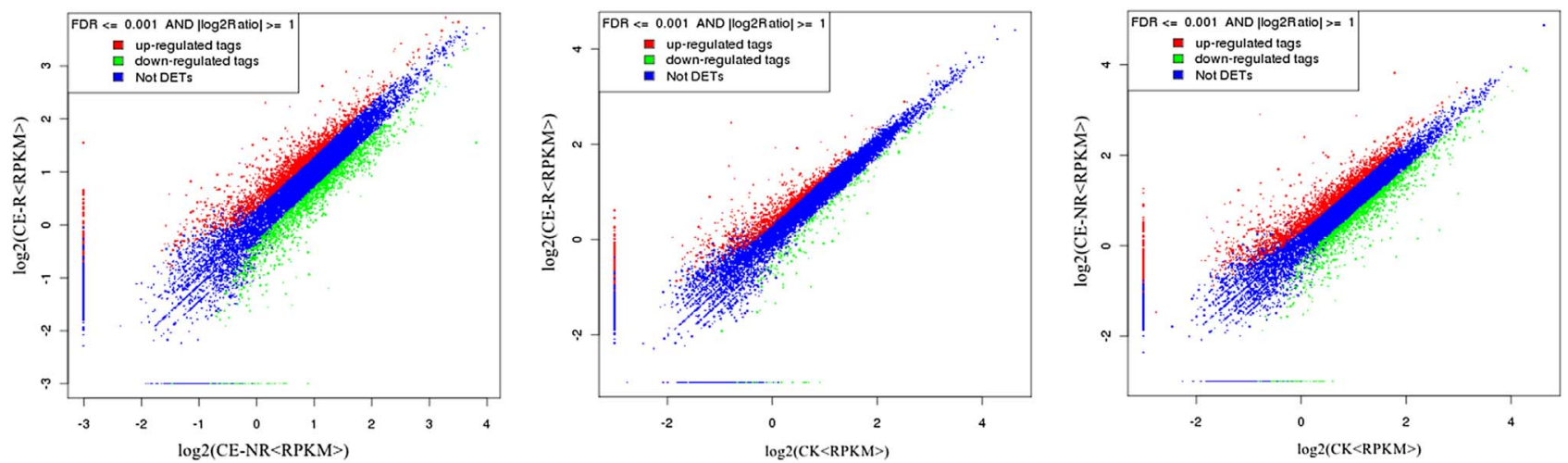

Figure 2. Scatter chart of differential expression genes between groups. Figure $2 \mathrm{~A}$ shows differential expression genes between the CE-R and CE-NR groups. Figure 2B shows differential expression genes between the CE-R and CK groups. Figure $2 \mathrm{C}$ shows differential expression genes between the CE-NR and CK groups. $X$-axis and $Y$-axis presents two samples log2 value of gene expression, and the expression was calculated using the RPKM method (reads per kilobase transcriptome per million mapped reads) as shown in section "Materials and methods", red (up) and green (down) dots mean the gene has significant differences (FDR $\leq 0.001$, two fold difference), and the blue dot means no significant differences.

\section{Discussion}

Based on previous studies, we speculated that the small intestine of Kazakh sheep with different MHC genotypes was the first line of defense against E. granulosus infection, and the immune response mechanism in the intestine might be related to disease resistance. The experimental results confirmed our hypothesis that DEGs related to innate immune response and adaptive immune response were found in different groups of sheep, some of which are worthy of attention. 
Table 3. Partial differential expression genes in the intestine, organized according to their function group.

\begin{tabular}{|c|c|c|c|}
\hline Gene & Gene ID & Description & $\begin{array}{c}\text { Fold change between } \\
\text { CE-R and CE-NR }\end{array}$ \\
\hline \multicolumn{4}{|c|}{ Epithelial barrier-lectin secretion } \\
\hline CLEC9A & 101119033 & C-type lectin domain containing 9A & 11.73 \\
\hline CLEC10A & 101117600 & C-type lectin domain containing $10 \mathrm{~A}$ & 3.1 \\
\hline CLEC4G & 101123627 & -type lectin domain containing $4 \mathrm{G}$ & 8.13 \\
\hline \multicolumn{4}{|l|}{ NK cell activation } \\
\hline IRAK1 & 101121405 & Interleukin-1 receptor-associated kinase 1 & 3.24 \\
\hline NKG7 & 101115817 & Natural killer cell granule protein 7 & 7.65 \\
\hline \multicolumn{4}{|c|}{ Mast cell activation and immune killing } \\
\hline MCP1 & 443546 & Monocyte chemoattractant protein-1 & 4.59 \\
\hline IRAK1 & 101121405 & Interleukin-1 receptor-associated kinase 1 & 3.24 \\
\hline \multicolumn{4}{|c|}{ T lymphocyte activation } \\
\hline NFATC2 & 101119239 & Recombinant nuclear factor of activated T cells, cytoplasmic 2 & 5.38 \\
\hline NFAT5 & 101113952 & Nuclear factor 5 of activated T cells & 2.93 \\
\hline \multicolumn{4}{|c|}{ Chemokine expression } \\
\hline CXCL12 & 101121145 & Chemokine (C-X-C motif) ligand 12 & 3.42 \\
\hline CXCL14 & 101105243 & Chemokine (C-X-C motif) ligand 14 & 4.61 \\
\hline CCL4 & 101114285 & Chemokine (C-C motif) ligand 4 & 2.88 \\
\hline CCL5 & 101115553 & Chemokine (C-C motif) ligand 5 & 10.24 \\
\hline CCL11 & 101119832 & Chemokine (C-C motif) ligand 11 & 5.41 \\
\hline CCL25 & 678679 & Chemokine (C-C motif) ligand 25 & 4.2 \\
\hline CCL28 & 780500 & Chemokine (C-C motif) ligand 28 & 4.48 \\
\hline \multicolumn{4}{|c|}{ Promote white blood cell migration } \\
\hline TICAM1 & 101111016 & Toll like receptor adaptor molecule 1 & 3.82 \\
\hline \multicolumn{4}{|c|}{ Compensatory inflammation repair mechanism } \\
\hline CYP17A1 & 493968 & Cytochrome P450, family 17 , subfamily A, polypeptide 1 & 15 \\
\hline CYP1A1 & 100170113 & Cytochrome P450, family 1 , subfamily A, polypeptide 1 & 5.9 \\
\hline CYP2C19 & 100534653 & Cytochrome P450, family 2, subfamily C, polypeptide 19 & 11 \\
\hline CYP3A24 & 100170111 & Cytochrome P450, family 3 , subfamily A, polypeptide 24 & 7.91 \\
\hline \multicolumn{4}{|c|}{ TNF expression related } \\
\hline TNFRSF13B & 101122200 & Tumor necrosis factor receptor superfamily member $13 \mathrm{~B}$ & 4.18 \\
\hline TNFRSF17 & 101111149 & Tumor necrosis factor receptor superfamily member 17 & 14.75 \\
\hline TNFRSF1A & 100135698 & Tumor necrosis factor receptor superfamily member $1 \mathrm{~A}$ & 3.03 \\
\hline TNFRSF4 & 101108407 & Tumor necrosis factor receptor superfamily member 4 & 2.88 \\
\hline TNFSF10 & 101114153 & Tumor necrosis factor receptor superfamily member 10 & 3.36 \\
\hline TNFSF13 & 101123054 & Tumor necrosis factor receptor superfamily member 13 & 3.13 \\
\hline TNFSF15 & 101102957 & Tumor necrosis factor receptor superfamily member 15 & 2.97 \\
\hline TNFSF18 & 101108765 & Tumor necrosis factor receptor superfamily member 18 & 5.15 \\
\hline \multicolumn{4}{|l|}{ MHC related } \\
\hline LOC101103957 & 101103957 & / & 6.11 \\
\hline LOC101108696 & 101108696 & I & 4.45 \\
\hline LOC101109747 & 101109747 & I & 3.38 \\
\hline LOC101120148 & 101120148 & I & 3 \\
\hline LOC101109080 & 101109080 & l & 12.71 \\
\hline LOC101105609 & 101105609 & I & 6.35 \\
\hline LOC101107908 & 101107908 & I & 4.92 \\
\hline
\end{tabular}

\section{Innate immune response plays the first defense role in CE resistance in sheep}

The innate immune system is the first line of defense for the host to protect itself against invasion and infection by various parasites. During the infection process, as important recognition receptors, lectins can bind with carbohydrate molecules on the surface of parasites, initiate related immune response, kill and eliminate invasive parasites, and play an important role in innate immunity against parasite infection.

In this study, we found that when Kazakh sheep were infected with oncospheres, the expression of CLEC9A,
CLEC1OA, and CLEC4G genes was significantly higher in the CE-R group than in the CE-NR group. All these genes are $\mathrm{C}$-type lectin receptor family members, which has been demonstrated for immunomodulatory effects against parasitic infections in the epithelial layer [45]. Reports have shown that upregulation of C-type lectin genes is beneficial to recognition of parasite antigens, thus activating antigen presentation more effectively [31], which has a positive effect on disease resistance $[18,49]$. Based on these reports and our findings, we speculated that the high regulation of lectin receptor genes in the intestinal tissue may enhance the recognition of parasite antigens and present them to the internal immune cells, which 


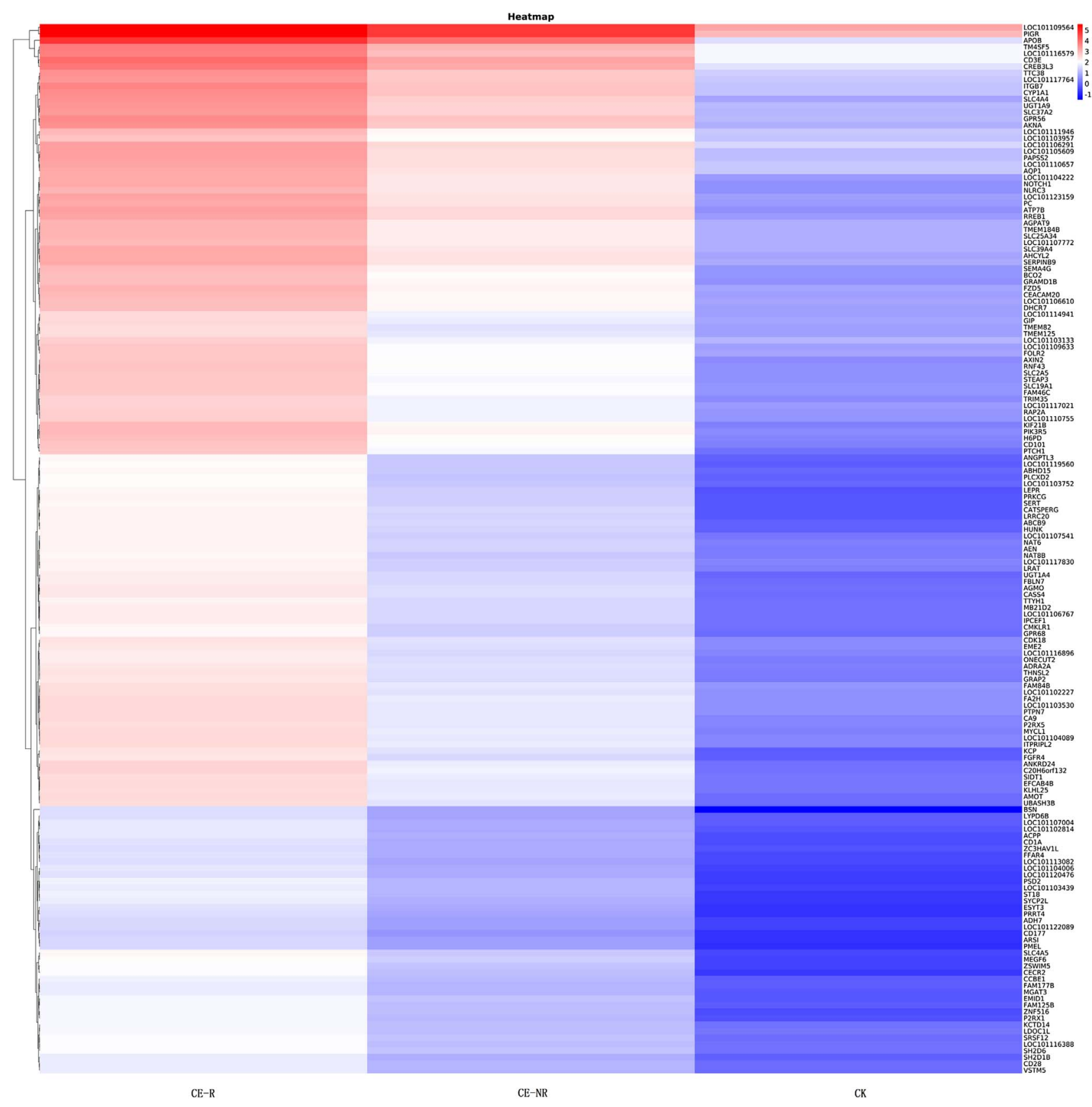

Figure 3. Heatmap of partial DEGs between sample groups.

can induce the immune system to kill the parasite. This effect was more significant in the CE-R sheep than in the CE-NR sheep, and it may lead to different E. granulosus resistance of Kazakh sheep with different MHC genotypes.

Furthermore, MHC class I chain-related protein A (MICA) is a natural ligand of C-type lectin like activated receptor, which is mainly expressed in the human intestinal epithelium. Cellular stress such as parasitic infections and inflammation can upregulate the expression of MICA [3]. However, although we found that CLEC9A and other genes were upregulated in CE-R group, no evidence showed corresponding MHC natural ligand. This should be studied further.

In addition to the antigen recognition system, innate immune cells, including natural killer (NK) cells [43], mast cells [5], and macrophages [8] can also be activated and produce immune response when host tissues are infected by parasites. Reports have shown that if the expression levels of genes related to the activation and receptor recognition of these cells are upregulated, this may promote phagocytosis [37], killing of parasites [43] and inflammatory response [20] of hosts, and result in stronger resistance to parasites.

Natural killer cells are responsible for activating the cytotoxic killing effect in infections, mainly to defend against early infections by viruses [34], bacteria [15], and parasites [41]. The killing effect of NK cells on target cells depends on intracellular interaction of activated and inhibitory receptors on the cell surface [10]. NKG7 (Natural killer cell granule protein 7) serves as an activating receptor for NK cells, which is associated with 

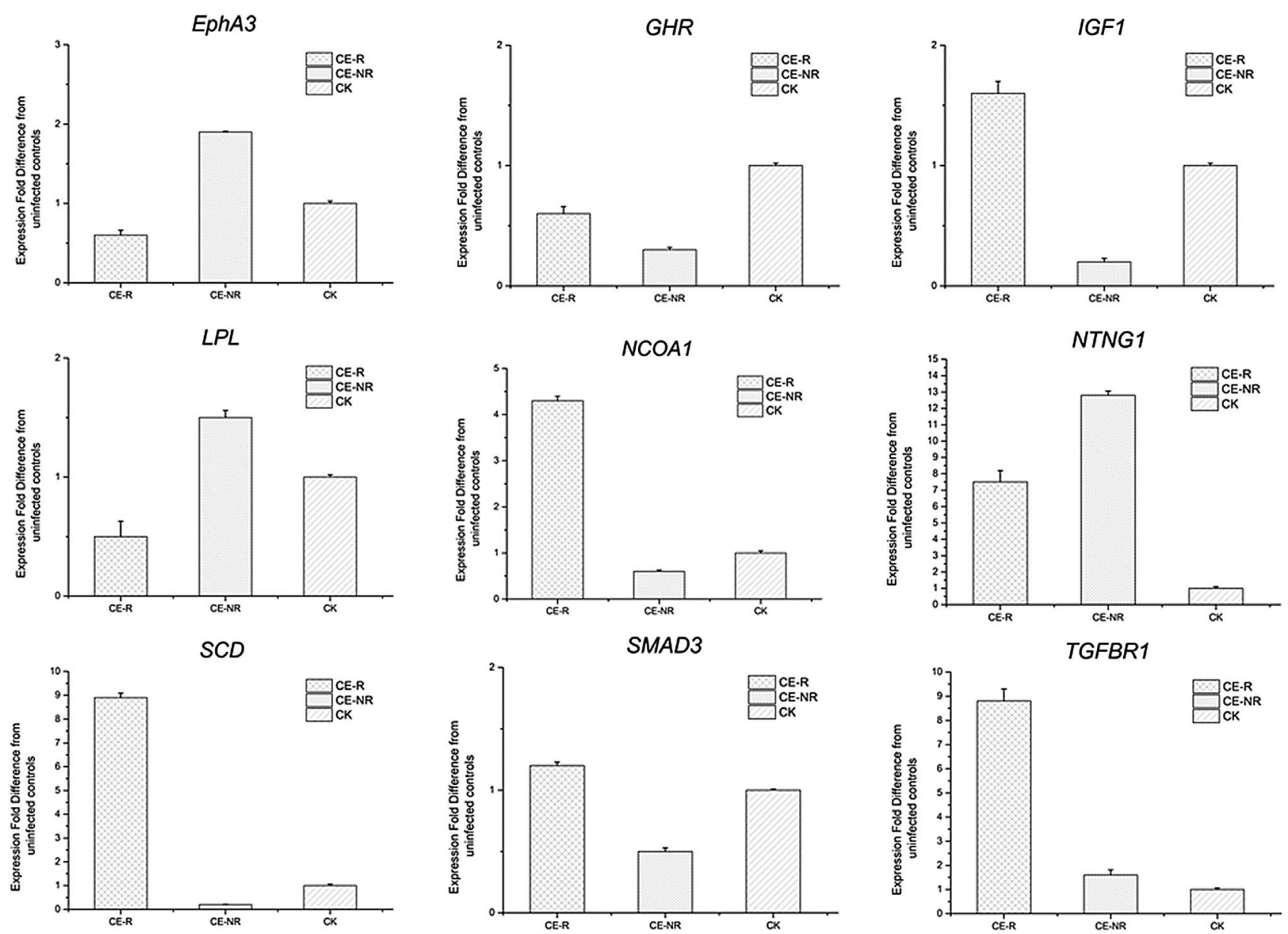

Figure 4. Validation of transcriptome data by qRT-PCR on nine randomly selected genes. Real-time PCR was employed to verify the regulation of genes detected by transcriptome. Nine differential expression genes were randomly selected. GAPDH was used as an internal control.

inflammatory diseases, since it could augment the production of inflammatory factors, including IFN- $\gamma$, responsible for type I immune responses [44]. In this study, the expression of $N K G 7$ was significantly higher in the CE-R group than in the CE-NR group, with a fold change of 7.65 ( $p$-value: $3.38 \mathrm{E}$ 211, FDR: 1.04E-209). A current study by Ng (personal communication) showed that $N K G 7$ was one of the most upregulated genes from the livers of mice infected with Leishmania donovani. Results indicated that NKG7 regulated the proinflammatory responses effectively, and the survival of the parasite was obviously affected [39]. Given these reports, and the results of our study, we inferred that the high expression of $N K G 7$ in CE-R sheep is beneficial for the host to activate NK cells and kill parasites, which is beneficial for CE resistance.

Mast cells are widely distributed around the capillaries under the skin and visceral mucosa, secreting a variety of cytokines, which participate in immune regulation (TB cell and APC cell activation), and express MHC and B7 molecules [16]. In our study, we found that mast cell-related genes, including FCRL6 and IRAK-1, were highly expressed in the CE-R group. IRAK-1 is related to Toll-like receptors (TLRs). When oncospheres of the parasite infect the small intestinal tissue, various antigens secreted by the parasite can be recognized by mast cells through TLRs, which activate effective immune response and produce immediate inflammation. This can be evidenced by the upregulation of genes related to inflammation and chemokines.

\section{Adaptive immune response played an important role in defense against $E$. granulosus infection}

It has been demonstrated that the mechanisms of host immune response to $\mathrm{CE}$ infection are complex. Response involves not only the innate immune system but also the adaptive immune system. In addition, we found that adaptive immune response played an important role in defense against E. granulosus infection. Nuclear factor of activated $\mathrm{T}$ cells (NFAT) could activate $\mathrm{T}$ cell response, thus the transcription of cytokines and other genes in immune response [24]. Besides T cells, NFAT is also expressed on many immune cells, such as B lymphocytes [23]. In this study, we found that the expression of NFATC2 was higher in the CE-R group than in the CE-NR group, with a 5.38 fold change ( $p$-value: 4.04E-51, FDR: 3.17E-50). Furthermore, the NFAT5 was upregulated in the CE-R group, with a 2.93 fold change ( $p$-value: 1.84E-27, FDR: 8.91E-27). Therefore, it was speculated that the high expression of NFAT-activated T cells, or expressed on B cells, induces adaptive immune response, and produces antibodies. 
As expected, in our study, we found that $\operatorname{IgM}$ and $\operatorname{IgE}$ were significantly more highly expressed in CE-resistant sheep than in non-resistant sheep.

\section{Metabolic changes during defense responses against $E$. granulosus infection in Kazakh sheep}

Finally, our study revealed that cytochrome metabolism may contribute to defense against E. granulosus infection. KEGG pathway enrichment analysis showed that many DEGs associated with Cytochrome 450 (CYP450), including CYP1A, CYP3A and CYP26A in CE-resistant sheep were clearly higher than in CE-non-resistant sheep. These DEGs were mainly enriched in the "metabolism of xenobiotics by cytochrome P450" pathway (46 genes, p-value: 2.357955e-08; Pathway ID: ko00980) and "drug metabolism - cytochrome P450" pathway (39 genes, $p$-value: 5.695932e-08, Pathway ID: ko00982). The CYP450 enzymes family includes membrane-bound hemoproteins that play a pivotal role in the detoxification of xenobiotics, cellular metabolism, and homeostasis [14]. It has been reported that genes of the CYP450 family varied similarly in the liver of experimental mice infected with $E$. multilocularis [30]. Moreover, a recent study demonstrated that members of the CYP family CYP1A could enhance inflammatory responses [48]. This aligns with the results of our previous study showing several upregulated miRNAs, which are related to NF-KB inflammation pathways, and play important roles in the response of intestinal tissue to E. granulosus infection in sheep with resistant haplotypes [22]. We can infer that the upregulation of the CYP family in the present study might enhance inflammatory responses, thus contributing more strongly to defense responses to $\mathrm{CE}$ infection in CE-R sheep than in CE-NR sheep.

In addition, we found that retinol metabolism is associated with CE infection in sheep. After CE infection, 38 DEGs associated with retinol metabolism, including $A D H$ [7], $R D H$ [50], UGT [25], and LRAT [35], were significantly higher in CE-resistant sheep than in non-resistant sheep. These genes are already known to be associated with the binding, metabolic process, and transport of retinol. Reports have shown that retinol plays an important role in maintaining host immune function. Cotton rats lacking retinol had a significantly higher infection rate of filarial worms compared with normal cotton rats [46], and a similar finding was reported in research of human infection with filarial worms [40]. Parasites may take retinol preferentially from the host at the infected site for their own development, differentiation, growth, and reproduction. High concentrations of retinol in worms have a significant effect on their growth rate. Other studies have suggested that retinol is critical to defense against parasite infections, and this is due to the fact that a lack of retinol damages the epithelial tissue barrier and helps filariae to penetrate and migrate in the host, and local immune response of the mucosal surface thereby decreases [47]. Retinol is, therefore, vital in host resistance to parasitic infections. Based on these findings, we speculated that the high expression of these genes in the CE-R group might play an important role in intestinal defense against E. granulosus infection, by hindering parasite damage to the epithelial tissue barrier, as well as penetration and migration to the host organ. These types of inhibitory effects might be related to the artificial infection resistance in the small intestinal tissue of Kazakh sheep reported by us previously [4, 5]. This is the first finding of a relationship between retinol metabolism and $\mathrm{CE}$ resistance in the host.

In conclusion, we found a number of genes related to the resistance of Kazakh sheep to echinococcosis in the present study, mainly related to lectin secretion, immune cell activation, antigen binding, chemokine expression, and metabolic pathways like retinol. Further studies, like using RNAi or Crispr/cas9 to verify the function of these genes are still needed in the future. In addition to transcriptomics analysis, Multi-omics research including proteomics, metabolomics, and lipidomics is still needed to reveal the molecular mechanism of CE resistance in Kazakh sheep with the resistant haplotype. We are currently carrying out a proteomics study to select different proteins in the intestinal tissue of these Kazakh sheep. Combined with the results of this study, we hope future results will enable us to provide a comprehensive explanation of resistance to $\mathrm{CE}$ in Kazakh sheep.

Acknowledgements. This work was supported by the National Natural Science Foundation of China (Grant Nos. 31260535 and 31402048).

\section{Conflict of interest}

The authors declare that they have no conflicts of interest in this work: we declare that we do not have any commercial or associative interest that represents a conflict of interest in connection with the work submitted.

\section{References}

1. Anand N, Kanwa R, Dubey M, Vahishta R, Sehgal R, Verma A, Kanwar J. 2015. Effect of lactoferrin protein on red blood cells and macrophages: mechanism of parasite-host interaction. Drug Design Development and Therapy, 9, 3821-3835.

2. Audic S, Claverie JM. 1997. The significance of digital gene expression profiles. Genome Research, 7(10), 986.

3. Bauer S, Groh V, Wu J, Steinle A, Phillips JH, Lanier LL, Spies T. 1999. Activation of NK cells and T cells by NKG2D, a receptor for stress-inducible MICA. Science, 285(5428), 727729.

4. Benjamini Y, Yekutieli D. 2005. False discovery rate-adjusted multiple confidence intervals for selected parameters. Journal of the American Statistical Association, 100(469), 71-81.

5. Bidri M, Vouldoukis I, Mossalayi MD, Debr P, Guillosson JJ, Mazier D, Arock M. 1997. Evidence for direct interaction between mast cells and Leishmania parasites. Parasite Immunology, 19(10), 475-483.

6. Bronnert J, Wulff A, Hillejan L, Reiter-Owona I. 2017. Pulmonary Echinococcus granulosus infection. Infection, 45 (4), 1-2.

7. Chase JR, Poolman MG, Fell DA. 2009. Contribution of NADH increases to ethanol's inhibition of retinol oxidation by human $\mathrm{ADH}$ isoforms. Alcoholism, Clinical and Experimental Research, 33(4), 571-580.

8. Coakley G, Harris NL. 2020. Interactions between macrophages and helminths. Parasite Immunology, 42(7), e12717. 
9. Conn DB, Swiderski Z. 2008. A standardised terminology of the embryonic envelopes and associated developmental stages of tapeworms (Platyhelminthes: Cestoda). Folia Parasitologica, 55 (1), 42-52.

10. Cooper MA, Fehniger TA, Fuchs A, Colonna M, Caligiuri MA. 2003. NK cell and DC interactions. Trends in Immunology, 25 (1), 47-52.

11. Du X, Hui W, Zhao B, Guo Y, Ma J, Ma S, Muyesaer B, Liu X, Jia B. 2012. Construction of subtracted library of small intestine in Kazakh sheep with two MHC-DRB1 genotype infected by Echinococcus oncosphere. Journal of Shihezi University (Natural Science), 30(5), 587-591.

12. Emrah E, Bora Ö, Fatih M, Serkan K, İzzet Ş. 2017. Molecular characterization of Echinococcus granulosus isolates obtained from different hosts. Mikrobiyoloji Bulteni, 51(1), 79-86.

13. Eng CHL, Lawson M, Zhu Q, Dries R, Koulena N, Takei Y, Yun J, Cronin C, Karp C, Yuan GC, Cai L. 2019. Transcriptome-scale super-resolved imaging in tissues by RNA seqFISH+. Nature, 568(7751), 235-239.

14. Feng S, He X. 2013. Mechanism-based Inhibition of CYP450: An indicator of drug-induced hepatotoxicity. Current Drug Metabolism, 14(9), 921-945.

15. Fink LN, Zeuthen LH, Christensen HR, Morandi B, Frøkiaer H, Ferlazzo G. 2007. Distinct gut-derived lactic acid bacteria elicit divergent dendritic cell-mediated NK cell responses. International immunology, 19(12), 1319-1327.

16. Frossi B, Mion F, Pucillo C. 2016. Deciphering new mechanisms on T-cell costimulation by human mast cells. European Journal of Immunology, 46(5), 1105-1108.

17. Garcia-Longoria L, Palinauskas V, Ilgunas M, Valkiunas G, Hellgren O. 2020. Differential gene expression of Plasmodium homocircumflexum (lineage pCOLL4) across two experimentally infected passerine bird species. Genomics, 112(4), 28572865.

18. Geijtenbeek T, Gringhuis S. 2009. Signalling through C-type lectin receptors: shaping immune responses. Nature Reviews Immunology, 9(7), 465-479.

19. Glass EJ, Jensen K. 2007. Resistance and susceptibility to a protozoan parasite of cattle-Gene expression differences in macrophages from different breeds of cattle. Veterinary Immunology and Immunopathology, 120(1), 20-30.

20. Gowda N, Wu X, Sanjeev K, Maria F, Channe G, Simona S. 2013. CD36 Contributes to malaria parasite-induced proinflammatory cytokine production and NK and T cell activation by dendritic cells. PlosOne, 8(10), e77604.

21. Hui W, Shen H, Jiang S, Jia B. 2012. MHC-DQB1 Variation and its association with resistance or susceptibility to cystic echinococcosis in Chinese Merino sheep. Asian-Australasian Journal of Animal Sciences, 25(12), 1660-1666.

22. Jiang S, Li X, Wang X, Ban Q, Hui W, Jia B. 2016. MicroRNA profiling of the intestinal tissue of Kazakh sheep after experimental Echinococcus granulosus infection, using a highthroughput approach. Parasite, 23, 23.

23. Khalid M, Hani A, Ralf M, Thuy PDA, Krisna M, Amiya KP, Andris A, Valesca B, Ugur S, Eisaku K, Stefan K-H, Edgar S. 2014. NF- $\kappa$ B factors control the induction of NFATc1 in B lymphocytes. European Journal of Immunology, 44(11), 33923402.

24. Lee N, Kim D, Kim W. 2019. Role of NFAT5 in the immune system and pathogenesis of autoimmune diseases. Frontiers in Immunology, 10, 270.

25. Li F, Fu C, Xie Y, Wang A, Li J, Gao J, Cui X. 2019. Transcriptional responses to starvation stress in the hepatopancreas of oriental river prawn Macrobrachium nipponense. Environmental Pollution, 252, 14-20.
26. Li R, Yu C, Li Y, Lam T-W, Yiu S-M, Kristiansen K, Wang J. 2009. SOAP2: an improved ultrafast tool for short read alignment. Bioinformatics, 25(15), 1966-1967.

27. Li RQ, Zhu HM, Ruan J, Qian WB, Fang XD, Shi ZB, Li YR, Li ST, Shan G, Kristiansen K, Li SG, Yang HM, Wang J, Wang J. 2010. De novo assembly of human genomes with massively parallel short read sequencing. Genome Research, 20(2), 265272.

28. Li RY, Hui WQ, Jia B, Shi GQ, Zhao ZS, Shen H, Peng Q, Lv LM, Zhou QW, Li HT. 2011. The relationship between MHCDRB1 gene second exon polymorphism and hydatidosis resistance of Chinese Merino (Sinkiang Junken type), Kazakh and Duolang sheep. Parasite, 18(2), 163-169.

29. Li RY, Jia B, Zhang WJ, Zhao ZS, Shi GQ, Shen H, Peng QA, Lv LM, Zhou QW, Du YC. 2010. Analysis of the relationship between MHC-DRB1 gene polymorphism and hydatidosis in Kazakh sheep. Asian-Australasian Journal of Animal Sciences, 23(9), 1145-1151.

30. Lin R, Lü G, Wang J, Zhang C, Xie W, Lu X, Mantion G, Martin H, Richert L, Vuitton DA, Wen H. 2017. Time course of gene expression profiling in the liver of experimental mice infected with Echinococcus multilocularis. PLoS One, 6(1), e14557.

31. Liu B, Li P, Yang G. 2015. The immunomodulatory role of c-type lectin receptors in parasitic infection. Chinese Journal of Parasitology \& Parasitic Diseases, 33(3), 228-232.

32. Liu C, Xu Y, Cadavid-Restrepo AM, Lou Z, Yan H, Li L, Fu B, Gray DJ, Clements AA, Barnes TS, Williams GM, Jia W, Mcmanus DP, Yang Y. 2018. Estimating the prevalence of Echinococcus in domestic dogs in highly endemic for echinococcosis. Infectious Diseases of Poverty, 7(1), 77.

33. Liu S, Zhou X, Hao L, Piao X, Hou N, Chen Q. 2017. Genomewide transcriptome analysis reveals extensive alternative splicing events in the protoscoleces of Echinococcus granulosus and Echinococcus multilocularis. Frontiers in Microbiology, 8, 929.

34. Liu Y, Zheng J, Liu Y, Wen L, Huang L, Xiang Z, Lam K, Lv A, Mao H, Lau YL, Tu W. 2018. Uncompromised NK cell activation is essential for virus-specific CTL activity during acute influenza virus infection. Cellular \& Molecular Immunology, 15(9), 827-837.

35. Marwarha G, Berry DC, Croniger CM, Noy N. 2014. The retinol esterifying enzyme LRAT supports cell signaling by retinol-binding protein and its receptor STRA6. FASEB Journal, 28(1), 26-34.

36. Mortazavi A, Williams BA, Mccue K, Schaeffer L, Wold B. 2008. Mapping and quantifying mammalian transcriptomes by RNA-Seq. Nature Methods, 5(7), 621-628.

37. Murta SM, Ropert C, Alves RO, Gazzinelli RT, Romanha AJ. 1999. In-vivo treatment with benznidazole enhances phagocytosis, parasite destruction and cytokine release by macrophages during infection with a drug-susceptible but not with a derived drug-resistant Trypansoma cruzi population. Parasite Immunology, 10(21), 535-544.

38. Neves LBD, Teixeira PEF, Silva S, Oliveira FBD, Garcia DD, Almeida FBD, Rodrigues-Silva R, Machado-Silva JR. 2017. First molecular identification of Echinococcus vogeli and Echinococcus granulosus (sensu stricto) G1 revealed in feces of domestic dogs (Canis familiaris) from Acre, Brazil. Parasites \& Vectors, 10(1), 28.

39. Ng SS, Rivera FDL, Yan J, Corvino D, Das I, Zhang P, Kuns R, Chauhan SB, Hou J, Li X, Frame TCM, Mcenroe BA, Moore E, Na J, Engel JA, Soon MSF, Singh B, Kueh AJ, Herold MJ, Oca MMD, Singh SS, Bunn PT, Aguilera AR, Casey M, Braun M, Ghazanfari N, Wani S, Wang Y, Amante FH, Edwards CL, Haque A, Dougall WC, Singh OP, Baxter AG, Teng MWL, Loukas A, Daly NL, Cloonan N, Degli-Esposti MA, Uzonna J, 
Heath WR, Bald T, Tey S-K, Nakamura K, Hill GR, Kumar R, Sundar S, Smyth MJ, Engwerda CR. 2020. The NK cell granule protein NKG7 regulates cytotoxic granule exocytosis and inflammation. Nature Immunology, 21(10), 1205-1218.

40. Nmorsi OPG, Ukwandu NCD, Egwunyenga OA, Anyanwu LC, Odike M. 2007. Vitamin A status and ocular lesions in some rural Nigerians with onchocerciasis. African Journal of Biotechnology, 6(11), 1293-1297.

41. O'brien K, Stacey S-C, Knoll LJ. 2011. Parasite-mediated upregulation of NK cell-derived gamma interferon protects against severe highly pathogenic H5N1 influenza virus infection. Journal of Virology, 85(17), 8680-8688.

42. Paul S, Ruiz-Manriquez LM, Serrano-Cano FI, Estrada-Meza C, Solorio-Diaz KA, Srivastava A. 2020. Human microRNAs in host-parasite interaction: a review. 3 Biotech, 10(12), 510.

43. Roland J, Soulard V, Sellier C, Drapier AM, Santo JPD, Cazenave PA, Pied S. 2006. NK cell responses to Plasmodium infection and control of intrahepatic parasite development. Journal of Immunology, 177(2), 1229-1239.

44. Schuster IS, Andoniou CE. 2020. NKG7 - regulating endosomal pathways? Immunology and Cell Biology, 98(10), 802804.

45. Silva A, Teixeira T, Teixeira S, Machado F, MaD Santos, Tomiosso TC, Tavares PCB, Brígido RTES, Martins FA, Silva NSDL, Rodrigues CC, Roque-Barreira MC, Mortara RA, Lopes DS, Vila VDMR, Claudio VDS. 2017. Galectin-3: a friend but not a foe during Trypanosoma cruzi experimental infection. Frontiers in Cellular and Infection Microbiology, 7, 463.
46. Storey DM. 1982. Vitamin A deficiency and the development of Litomosoides carinii (Nematoda, Filarioidea) in cotton rats. Zeitschrift für Parasitenkunde, 67(3), 309-315.

47. Taylor V, Velásquez C, Burgos L, Carmona J, Correa A, Maestre A, Uscátegui R. 2009. Retinol levels, iron status, malaria and intestinal parasites: TH1/TH2 cytokines relationship. Revista Colombia Médica, 39(3), 276-286.

48. Tian LX, Tang X, Zhu J, Luo L, Ma X, Cheng S, Zhang W, Tang W, Ma W, Yang X, Lv C, Liang H. 2020. Cytochrome P450 1A1 enhances inflammatory responses and impedes phagocytosis of bacteria in macrophages during sepsis. Cell Communication and Signaling, 18(1), 70.

49. Wang S, Hu D, Wang C, Tang X, Du M, Gu X, Suo J, Min H, Fang R, Zhu X, Zhang X, Du A, Suo X, Liu X. 2018. Transcriptional profiling of innate immune responses in sheep PBMCs induced by Haemonchus contortus soluble extracts. Parasites \& Vectors, 25, 182.

50. Yu YS, Wang LL, Shen Y, Yap MKH, Yip S, Han W. 2010. Investigation of the association between all-trans-retinol dehydrogenase (RDH8) polymorphisms and high myopia in Chinese. Journal of Zhejiang University-Science Biomedicine \& Biotechnology, 11(11), 836-841.

51. Zheng H, Zhang W, Zhang L, Zhang Z, Li J, Lu G, Zhu Y, Wang Y, Huang Y, Liu J, Kang H, Chen J, Wang L, Chen A, Yu S, Gao Z, Jin L, Gu W, Wang Z, Zhao L, Shi B, Wen H, Lin R, Jones MK, Brejova B, Vinar T, Zhao G, Mcmanus DP, Chen Z, Zhou Y, Wang S. 2013. The genome of the hydatid tapeworm Echinococcus granulosus. Nature Genetics, 45(10), $1168-1175$.

Cite this article as: Li X, Jiang S, Wang X \& Jia B. 2021. Intestinal transcriptomes in Kazakh sheep with different haplotypes after experimental Echinococcus granulosus infection. Parasite 28, 14.

Reviews, articles and short notes may be submitted. Fields include, but are not limited to: general, medical and veterinary parasitology; morphology, including ultrastructure; parasite systematics, including entomology, acarology, helminthology and protistology, and molecular analyses; molecular biology and biochemistry; immunology of parasitic diseases; host-parasite relationships; ecology and life history of parasites; epidemiology; therapeutics; new diagnostic tools.

All papers in Parasite are published in English. Manuscripts should have a broad interest and must not have been published or submitted elsewhere. No limit is imposed on the length of manuscripts.

Parasite (open-access) continues Parasite (print and online editions, 1994-2012) and Annales de Parasitologie Humaine et Comparée (1923-1993) and is the official journal of the Société Française de Parasitologie. 